handlung ron nässenden und juckenden Derma tosen. Nr. 10. p. 291.

Luftduschen mit trockener Luft von Zimnertemperatur, alle Tage oder jeden 2. Tag $1 / 2$ Stunde lang mit $1 / 1$ St. Pause übten einen günstigen austrocknenden, schmerz- und juckstillenden, die Epidermisierung anregenden Effekt auf Ulcera cruris, nässende Ekzeme und juckende Dermatosen (Pruritus vulvae, Dermatitis herpetiformis) aus. Der Apparat "Siccor" wird von Reiniger, Gebbert \& Schall hergestellt und kann an jede elektrische Leitung angeschlossen werden.

Rudolf Krösing (Stettin).

\title{
The British Journal of Dermatology.
}

August 1913.

Corbett, Dudley. Eine Methode zur Standardisierung der von der Sabouraud-Noiréschen Pastille gelieferten Farben. pag. 249.

Corbett berichtet über farbenanalytische Experimente, die er an Sabouraud-Noiréschen Tabletten zum Zwecke der Standardisierung dieser Tabletten mit Lovibonds Tintometer angestellt. Seine Versuche ergaben, da $\beta$ die nicht den Röntgenstrablen exponierte Tablette eine Mischung von gelb und blau ist. Das blau verschwindet nach und nach, bis bei einem Punkte, der nnter $1 / 2$ Erythemdosis liegt, nur noch gelb in der Tablette vorhanden ist. Dann erscheint rot in wachsender Proportion, und verursacht eine Vertiefung des Farbentons. Das Verhältnis des gelbs bleibt immer konstant. Der experimentelle Fehler für rot beträgt etwa $0 \cdot 15$. Die von dem Autor benützte Standardfärbung für Tint $B$ ist eine Mischung von $15 \cdot 0$ gelb und 1.5 rot.

Oliver, W. Jenkins. Ein Fall ron Purpura nach Trauma. pag. 253.

Der Patient Olivers hatte sich beim Fußballspiel eine Knieverletzung zugezogen. An dieser Stelle kam es später zu einer Schwellung und zu einem Ausbruch von Purpura an dem kranken Bein. Die Purpura war ausschließlich auf das linke Bein unterhalb des Knies beschränkt.

$$
\text { Fritz Juliusberg (Posen). }
$$

\section{The Journal of cutan. diseas. incl. Syph.} XXXI. 1913. Nr. 5-7.

Winfield, James Mac Farlane. Soll Dermatologie i m dritten oder vierten Jahre des Medizinstudiums gelernt werden. pag. 305 . 